\title{
An Effective Technique for Simultaneous Indirect-Direct Teeth Traction Using Temporary Anchorage Device
}

\section{Rahma ElNaghy ${ }^{1,2 *}$ and Majd Hasanin ${ }^{1}$}

${ }^{1}$ University of Detroit Mercy, School of Dentistry, Orthodontic Department, Detroit, MI, USA

${ }^{2}$ Faculty of Dentistry, Nahda University in Beni-Suef (NUB), Egypt

${ }^{*}$ Corresponding author: Rahma ElNaghy BDS, MS, MOrthRCSEd, FCFO, Assistant Professor, Director of the Craniofacial Division Orthodontic Department, School of Dentistry, University of Detroit Mercy, Michigan, 2700 Martin Luther King Jr. Blvd. Office number 322, Detroit, MI 48208, USA; Fax: +1 (313) 494-6605; Tel: +1 (216) 971-2819; E-mail: elnaghra@udmercy.edu

Received: May 27, 2021; Accepted: June 10, 2021; Published: June 15, 2021

\section{Abstract}

Since the introduction of Temporary Anchorage Devices (TADs) in the orthodontic field, they have been proven to be versatile and multi-applicable in the management of various orthodontic situation. Here, we highlight the viability of simple technique using TADs for an effective and time-efficient space closure.

Text

Temporary anchorage devices (TADs) are widely incorporated in the orthodontic field. TADs can be utilized directly or indirectly for anchorage, intrusion, distalization and retraction. Traditionally, when TADs are used indirectly for teeth retraction, the anchored tooth is maintained in place by using a stainless-steel ligature wire from the tooth to the TAD, allowing the retracted tooth to slide along the arch wire using an active open coil spring against the anchored tooth, utilizing push mechanics for retraction. While in direct teeth retraction, TADs can be utilized for pull mechanics [1].
Introduced here is an effective approach for simultaneous indirect-direct retraction using TAD. In this technique; instead of ligating the anchored tooth to the TAD, the ligature wire is ligated on the arch wire instead while the open coil spring is active. This will allow the retracted tooth to slide along the arch wire without the need to keep an anchored tooth in place and delay its retraction. Moreover, the adjacent tooth can be directly retracted using an elastomeric power chain attached from the TAD to the tooth. Thus, providing a simultaneous indirect-direct tooth retraction by utilizing push-pull mechanics on different teeth at the same time (Figures 1 and 2).

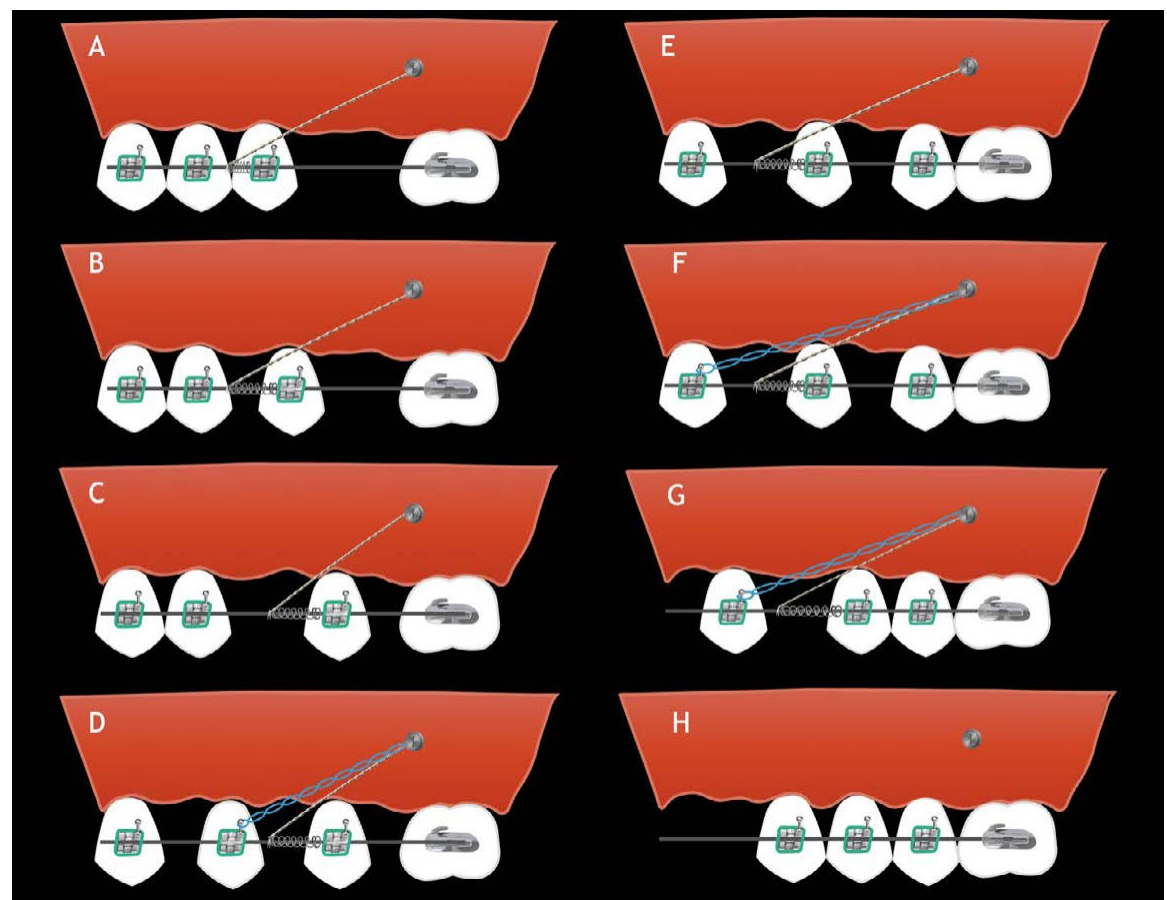

Figure 1: An illustration showing the application of TAD for simultaneous indirect-direct teeth retraction by utilizing push-pull mechanics on different teeth at the same time. 


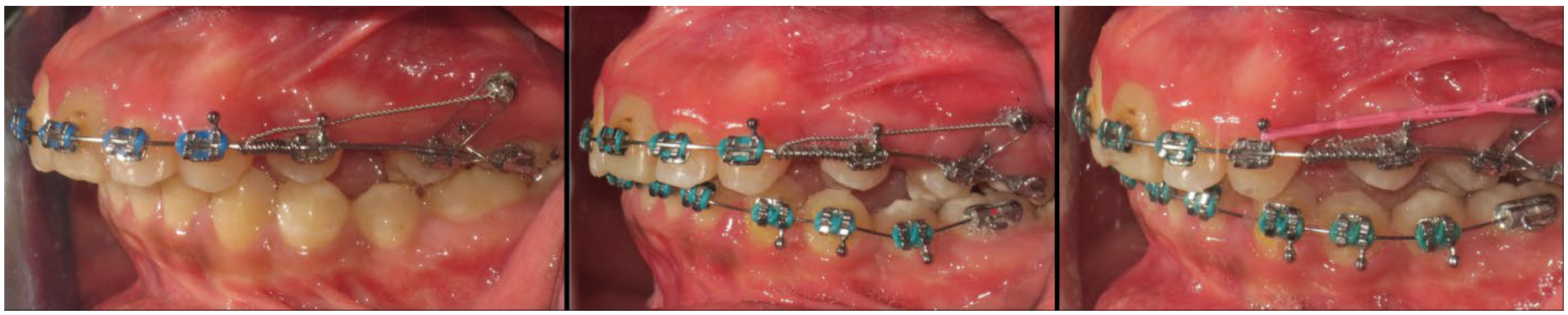

Figure 2: Clinical application of the technique.

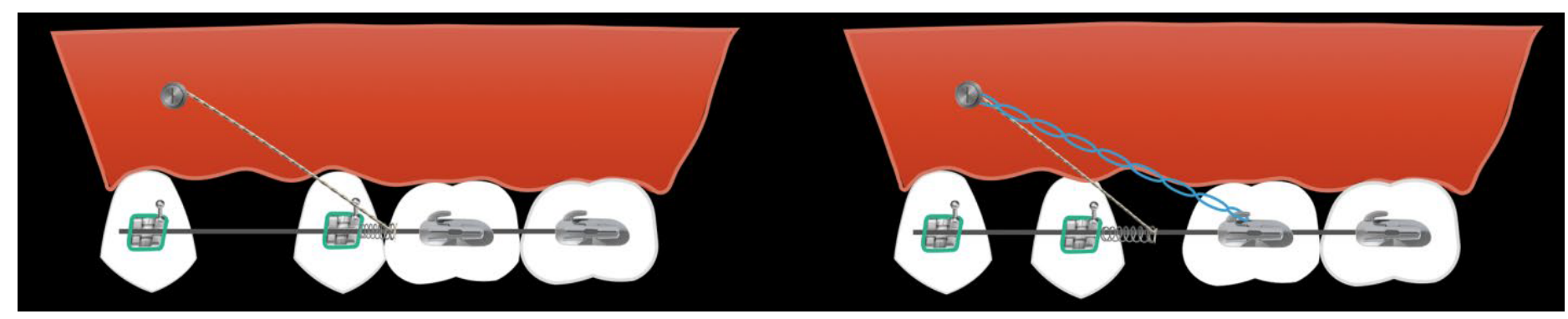

Figure 3: An illustration showing modification of the technique to be used for teeth protraction.

Additionally, the neighboring teeth can follow the retraction and exhibit lateral movement as a result of recoiling of the trans-septal fibers between the retracted and adjacent teeth, allowing driftodontics to take place. This technique can be modified for retraction or mesialization of the teeth (Figure 3).

\section{Reference}

1. Antoszewska-Smith J, Sarul M Łyczek J, Konopka T, Kawala B (2017) Effectiveness of orthodontic miniscrew implants in anchorage reinforcement during en-masse retraction: A systematic review and meta-analysis. Am J Orthod Dentofacial Orthop 151: 440-455. [crossref]

\section{Citation:}

ElNaghy R, Hasanin M (2021) An Effective Technique for Simultaneous Indirect-Direct Teeth Traction Using Temporary Anchorage Device. J Dent Maxillofacial Res Volume 4(2): 1-2. 